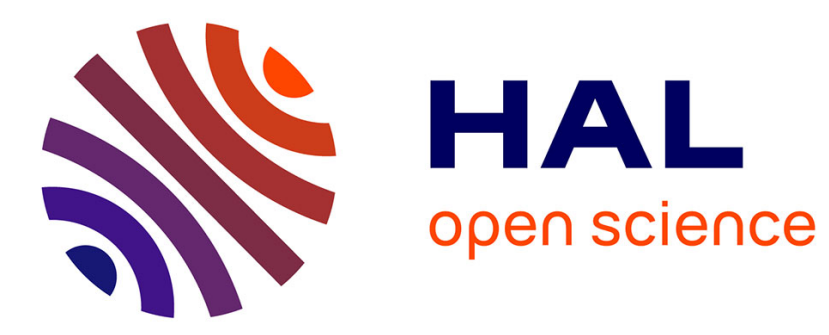

\title{
Towards measuring states of epistemic curiosity through electroencephalographic signals
}

\author{
Aurélien Appriou, Jessy Ceha, Smeety Pramij, Dan Dutartre, Edith Law, \\ Pierre-Yves Oudeyer, Fabien Lotte
}

\section{- To cite this version:}

Aurélien Appriou, Jessy Ceha, Smeety Pramij, Dan Dutartre, Edith Law, et al.. Towards measuring states of epistemic curiosity through electroencephalographic signals. IEEE SMC 2020 - IEEE International conference on Systems, Man and Cybernetics, Oct 2020, Toronto / Virtual, Canada. hal-02971795

\section{HAL Id: hal-02971795 \\ https://hal.inria.fr/hal-02971795}

Submitted on 19 Oct 2020

HAL is a multi-disciplinary open access archive for the deposit and dissemination of scientific research documents, whether they are published or not. The documents may come from teaching and research institutions in France or abroad, or from public or private research centers.
L'archive ouverte pluridisciplinaire HAL, est destinée au dépôt et à la diffusion de documents scientifiques de niveau recherche, publiés ou non, émanant des établissements d'enseignement et de recherche français ou étrangers, des laboratoires publics ou privés. 


\section{Towards measuring states of epistemic curiosity through electroencephalographic signals}

\author{
$1^{\text {st }}$ Aurélien Appriou \\ Inria, LaBRI (CNRS/Univ. Bordeaux /INP) \\ Talence, France \\ aurelien.appriou@inria.fr \\ $5^{\text {th }}$ Edith Law \\ University of Waterloo \\ Waterloo, Canada \\ $6^{\text {th }}$ Pierre-Yves Oudeyer \\ Inria \\ Talence, France
}

\author{
$2^{\text {nd }}$ Jessy Ceha \\ University of Waterloo \\ Waterloo, Canada
}

\author{
$3^{\text {rd }}$ Smeety Pramij \\ Inria \\ Talence, France
}

\author{
$4^{\text {th }}$ Dan Dutartre \\ Inria \\ Talence, France
}

\author{
$7^{\text {th }}$ Fabien Lotte \\ Inria, LaBRI (CNRS/Univ. Bordeaux /INP) \\ Talence, France
}

\begin{abstract}
Understanding the neurophysiological mechanisms underlying curiosity and therefore being able to identify the curiosity level of a person, would provide useful information for researchers and designers in numerous fields such as neuroscience, psychology, and computer science. A first step to uncovering the neural correlates of curiosity is to collect neurophysiological signals during states of curiosity, in order to develop signal processing and machine learning (ML) tools to recognize the curious states from the non-curious ones. Thus, we ran an experiment in which we used electroencephalography (EEG) to measure the brain activity of participants as they were induced into states of curiosity, using trivia question and answer chains. We used two ML algorithms, i.e. Filter Bank Common Spatial Pattern (FBCSP) coupled with a Linear Discriminant Algorithm (LDA), as well as a Filter Bank Tangent Space Classifier (FBTSC), to classify the curious EEG signals from the non-curious ones. Global results indicate that both algorithms obtained better performances in the 3-to-5s time windows, suggesting an optimal time window length of 4 seconds $(63.09 \%$ classification accuracy for the FBTSC, 60.93\% classification accuracy for the FBCSP+LDA) to go towards curiosity states estimation based on EEG signals.
\end{abstract}

Index Terms - curiosity - mental state - learning - ElectroEncephaloGraphy - passive Brain Computer Interfaces

\section{INTRODUCTION}

Curiosity is an important mental state associated with spontaneous exploration, active learning, facilitated memorization and sustained engagement [1]. Recent research in psychology [2] and neuroscience [3] has shown its pervasive role across multiple dimensions of human cognition and learning. The design of robotic and computer architectures can also be guided by our understanding and conceptualizations of curiosity [4], [5]. Human-computer interaction (HCI) researchers have investigated how systems can elicit curiosity in order to inform the design of persuasive, engaging, and playful interactions [6], or to motivate and incentivize crowd workers [7]. Finally, as curiosity-driven learning has been argued to be a crucial feature for efficient education [1], [8], learning technologies are being developed to promote curiosity and motivate curiosity-driven behaviours in students [9].

Brain-Computer Interfaces (BCIs) enable users to interact with computers by using brain activity, as measured by elec- troencephalography (EEG) signals [10]. There are two types of BCIs: active BCIs, where brain activity is converted into a command to an application, and passive BCIs, where the goal is to monitor the user's mental state in real time and adapt the interaction accordingly [11].

The premise of this paper is that passive BCIs that can monitor, through neurophysiological signals, the level of curiosity of users can be both a useful tool for understanding curiosity, and beneficial to designers of interactive systems, who wish to adapt the interaction paradigm or application content to users' levels of curiosity. In the context of active BCIs, which are known to be notoriously difficult for novices to use [12], it would be beneficial to adapt the BCI training tasks to the mental states of these users, e.g., their curiosity level, and adapt the BCI tasks to users' interests in order to prevent boredom and improve learning. In this work, we conducted an experiment in which subjects were given a trivia questionbased task, designed to elicit different levels of curiosity. We used signal processing and machine learning tools to analyze the EEG signals collected through this protocol, and assessed how well we can estimate curiosity through EEG signals using Machine Learning (ML) classifiers. Results show that F1 score can reach $63 \%$ from only a few seconds of EEG, depending on the pre-processing and ML algorithms chosen.

The first to propose a definition of curiosity was William James, who said that "curiosity is an instinct that evolved to facilitate survival and adaptation through active exploration of the environment" [13]. Daniel Berlyne then introduced a multi-dimensional model of curiosity, characterizing this psychological state in two dimensions, i.e., perceptual/epistemic curiosity, and specific/diversive curiosity [14]. Following this theory, perceptual curiosity refers to "a drive which is aroused by novel stimuli and reduced by continued exposure to these stimuli", whereas epistemic curiosity refers to "a desire to acquire knowledge, and applies mainly to humans" [15]. On the second dimension, specific curiosity is defined by the desire for a particular piece of information, whereas diversive curiosity is defined as a more general seeking for stimulation that is related to boredom. Although no scientific consensus 
has been reached concerning the definition of curiosity, certain types of stimuli are known to trigger it, i.e., those of surprising, novel, or intermediate complexity, as well as activities that are characterized by a knowledge gap or errors in prediction [1]. Such triggers can lead to different momentary states of curiosity, including epistemic [16], [17] and perceptual curiosities [13]. Epistemic curiosity has been particularly studied through psychological experiments in the last decades [18], and has more recently been the object of neuroscientific experiments.

Several studies have been run to better understand the neural mechanisms underlying states of curiosity. In [19], the authors scanned participants with functional magnetic resonance imaging (fMRI) in order to study the brain areas activated during the triggering of curiosity states with trivia questions. They observed that the curiosity induced by the trivia questions was correlated to brain activity in the caudate region, an area shown to be associated with anticipated rewards [20]. Moreover, they also found a correlation between surprising new information and activation of brain areas linked to memory. In [21], the question was raised as to whether curiosity enhances longterm memory, similar to the way anticipated rewards do. They likewise conducted a fMRI study with trivia question tasks, and found a correlation between curiosity levels and variations of activation in the right hippocampus and bilateral nucleus accubens, both involved in long-term memory improvements [13]. Using frontal EEG asymmetry-a common tool for measuring engagement and motivation - [22] investigated the relation between curiosity and learning. Participants performed trivia question tasks, similar to those used in [19], [21], while the EEG signals from the frontal cortex were recorded. Here, researchers found a correlation between frontal brain asymmetry (FBA) and memory recall; however they did not observe any correlation between FBA and self-reported curiosity.

In summary, these neuroscientific studies, mainly based on fMRI, support the existence of a correlation between epistemic curiosity and memory/learning, as well as correlations between states of curiosity and activation in specific brain regions. However, these prior works did not perform continuous state monitoring. In this work, we propose to measure states of epistemic curiosity using EEG. Compared to fMRI, which can be expensive and difficult to use outside of the lab, EEG provides a usable, portable and affordable tool for measuring the temporal activation of brain states associated with curiosity, making it suitable for applications such as BCIs.

\section{Materials \& Methods}

We describe in detail the experimental setup, including the participants involved, the protocol, the signal pre-processing procedure, the EEG-based curiosity classifiers used, and the evaluations performed.

\section{A. Participants}

Twenty-seven participants $(\mathrm{N}=27)$ were recruited through ads posted on social media and the local university mailing lists $(13 \mathrm{~F} / 14 \mathrm{M}$; aged $28.7 \pm 4.0)$. Levels of education varied between high school diploma and Ph.D. To be included in the experiment, people had to be at least 18 years old, speak French, and consent to the study ${ }^{1}$. Non-inclusion criteria include bad vision, heart condition, neurological or psychological diseases, and emotion-related problems. Finally, as per typical EEG studies protocol, we asked participants not to drink coffee or tea within the 2 hours prior to the experiment.

\section{B. Protocol}

Each participant participated in a single session that took place at Inria Bordeaux Sud-Ouest, lasting approximately 2 hours. Participants were asked to fill-in a pre-session questionnaire, assessing personal characteristics (such as gender, age and education). Next, EEG and ElectroOculoGram (EOG) electrodes were placed on the participants. For recordings, we used a BrainProduct ActiCHamp amplifier (EEG, 61 active electrodes in a 10/20 system, and EOG, 3 active electrodes to measure ocular artifacts). To our knowledge, except for [22] where they attempted - but did not succeed - to measure curiosity with electrodes placed onto the scalp recovering the frontal cortex, there has been no study seeking to classify curiosity levels from EEG signals. Due to the exploratory nature of our study, we covered the entire scalp with a relatively high EEG spatial resolution (61 electrodes). EEG signals were recorded and visually inspected using OpenViBE [23]. Sensors for ElectroDermal Activity (EDA), breathing and heart rate (HR) were also installed; though these signals were not used in the present study.

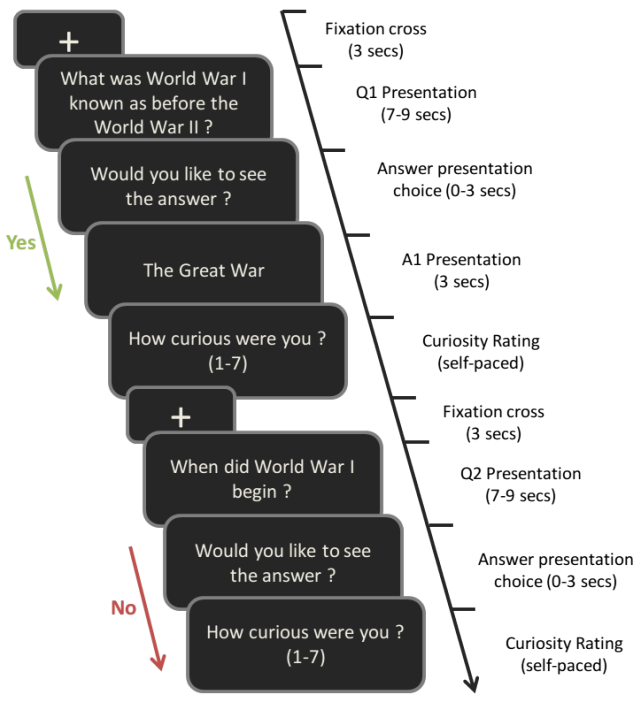

Fig. 1. Experiment flow: 1) fixation cross 2) question presentation 3) choice to reveal the answer 4) answer presentation 5) curiosity rating.

Following the setup, a 3 minute baseline, consisting of measuring EEG signals from participants at rest with opened eyes, was recorded. Finally, participants were asked to perform 4 runs of curiosity tasks (described below), around 10 minutes each, with 5 minute breaks between them. Each run consisted

\footnotetext{
${ }^{1}$ This study was approved by the ethics committee of Inria Bordeaux SudOuest (COERLE approval number 2019-13).
} 
of a series of trivia questions and answers, inducing different levels of curiosity. Before each question, a fixation cross was presented on the screen for 3 seconds, in order to get the participant ready. Figure 1 illustrates the experiment flow.

\section{Materials}

Prior work using trivia questions to elicit curiosity presented participants with question/answer pairs that did not have any link to previously viewed or future questions [19], [21], [22]. In our study, we introduce a novel protocol consisting of chains of trivia question/answer pairs-i.e., if participants were curious about the answer to a certain question, the following question would follow on the same topic. The assumption is that if participants were curious about a certain question, it was likely they would be curious about this topic in general. This new method allowed us to: 1) record a large enough amount of EEG signals from both curious and non-curious states, to then be able to train the curiosity classifiers with a balanced set of EEG examples, and 2) check the assumption that curiosity could be a mental state that increases over time, in the same way intrinsic motivation and self-directed learning increase when time spent in flow state increases [24].

The trivia questions used to elicit curiosity in this study came from an online trivia question dataset (https://www. randomtriviagenerator.com). The questions were grouped following a two-level categorization system. Questions from the website were already classified into classical trivia categories (referred to as first-order categories), such as Science, History, Geography, Arts, General Knowledge and Sports. We further classified the questions in each first-order category into groups of 4 to 20 questions based on 800 extracted keywords from the first-order category questions. For example, in the Geography category, we identified a sub-category based on the keyword "Nile". All questions from the Geography category with the word "Nile" were then grouped into a single chain of questions. Figures 1 and 2 show another example of a question chain based on the keyword "World War One".

All chains containing less than 4 or more than 20 questions were not used in this study. A minimum of 4 was chosen in order to have enough questions to define a subcategory. Conversely, we chose 20 as the maximum to ensure the subcategory was not too vague. Overall, the dataset consisted of 2000 questions/answers divided between 250 subcategories.

\section{Curiosity Task}

The curiosity task was presented on a computer screen using images - black background with white text. Each run was set up as follows: a first question from a random chain from a random category was displayed to the participant - for 7 to 9 seconds - right after a 3 -second fixation cross. The participant then had 2-3 seconds to choose to display the answer or not, as presented in Figure 1. The display time was determined based on the length of the question or answer in terms of number of characters. The participant chooses to display the answer by tapping on the keyboard space bar: this question

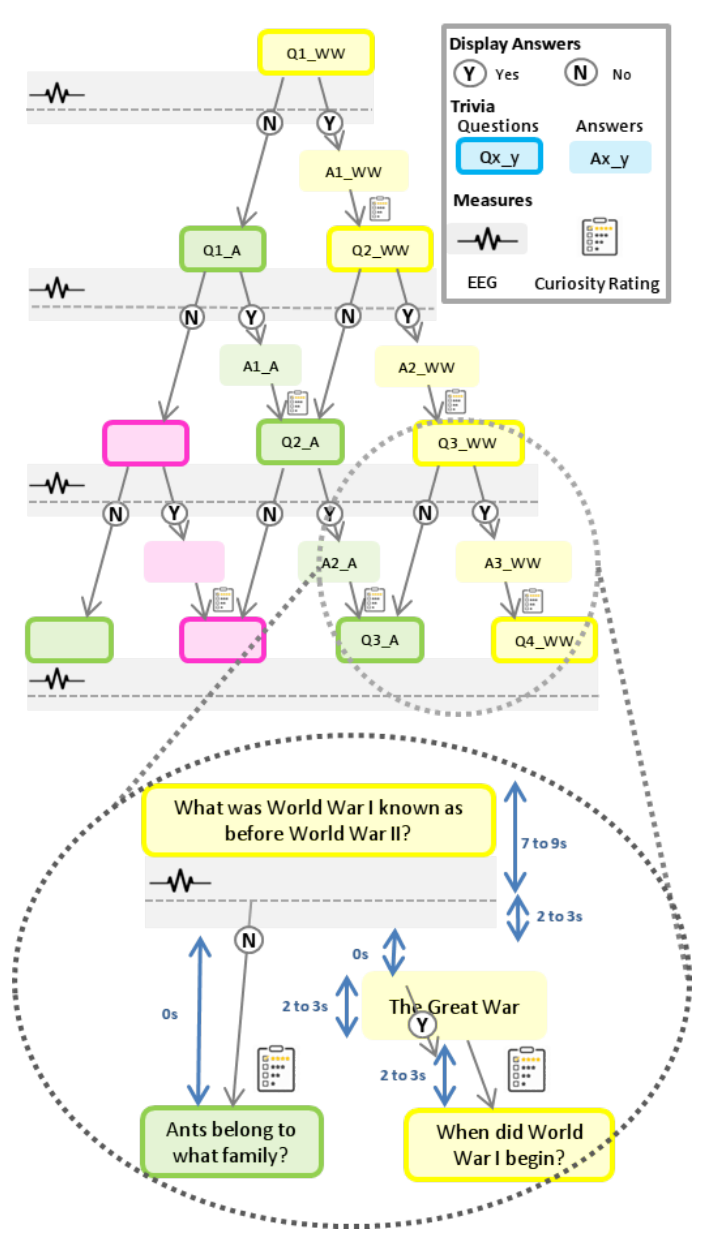

Fig. 2. The trivia questions/answers system. In the example, a question about World War One is presented: if they choose to display the answer, they will stay on the "World War One" topic, but will continue on another topic - here scientific questions about ants - if they skip the answer.

was then flagged as "curious", the concerned subcategory was considered interesting for the participant, and the answer was directly displayed on the screen for a few seconds. Participants were asked to rank their level of curiosity for the question on a 1-7 scale using a number pad right after the answer had been displayed. Following the rating, a new question randomly selected from the same subcategory was displayed.

If the participant did not choose to display the answer (by tapping on the keyboard) before the decision time elapsed, the question was labeled as "non-curious", the subcategory was not considered to be interesting to the participant. The curiosity rating scale was immediately administered without revealing the answer, followed by a fixation cross and a new random question from the next subcategory. A run ended only if at least 15 trials with questions marked as curious and 15 trials with questions marked as non-curious had been displayed. Thus, for each participant, we obtained at least 30 trials per run, i.e., a total of 120 trials in 4 runs: 60 trials per state of curiosity (curiosity \& no curiosity). 


\section{E. EEG signal processing \& classification}

Our system aims to discriminate curious from non-curious states using EEG signals. To do so, we employed machine learning approaches based on state-of-the-art algorithms developed for BCIs [25]-[27].

1) EEG Pre-processing: We first pre-processed EEG signals into $\mathrm{N}$-second windows, in order to create 5 different data sets, with 1-, 2-, 3-, 4- and 5-second windows. The EEG signals for each 10 minute run were divided into approximately 30 trials, i.e., one trial per trivia question displayed. More precisely, an EEG trial was defined as ending at the time the question disappeared from the screen, and starting $\mathrm{N}$-seconds earlier, as represented by the blue arrows on Figure 3. Note that no artifact removal algorithm has been used in this study.

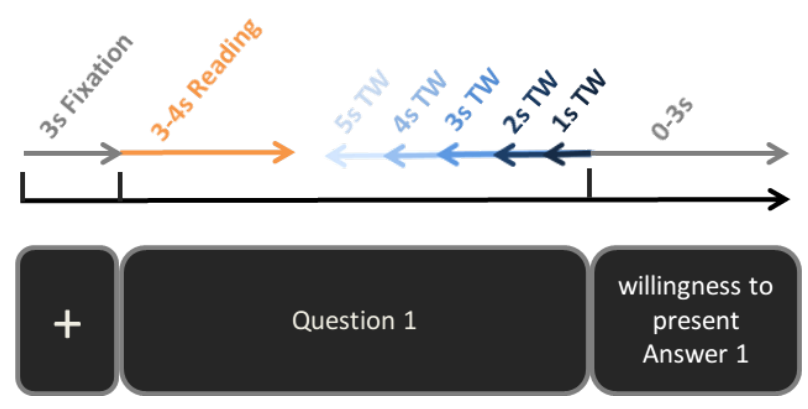

Fig. 3. Diagram representing the way we epoched the signals into 1, 2, 3, 4 and 5-seconds time windows (TW).

2) Classification Labels: Each trial was flagged as "answer" or "no-answer", based on the participant's choice to reveal the answer or not, respectively. Based on the flags and participants' self-reported curiosity ratings, we labeled each trial as "curious" if the trial was flagged as "answer" and the rating was higher than the mean of the participant's ratings; otherwise as "non-curious", if the trial was flagged as "no-answer" and the rating was lower than the mean of the participant's ratings. We obtained around 50 trials per curiosity level per participant (mean number of trials in class "non-curious" $=50.18 \pm 12.58$; mean number of trials in class "curious" = 46.85 \pm 12.11 ).

3) Machine Learning Algorithms: We used two ML algorithms that are exploring multiple frequency bands: (1) a Filter Bank Common Spatial pattern (FBCSP) coupled with a Linear Discriminant Analysis (LDA) [26], and (2) a Filter Bank Tangent Space Classifier (FBTSC) [27]. Both algorithms proved effective for mental state classification from EEG [27].

Prior work studying curiosity through EEG signals [22], only used two electrodes that were placed on the frontal cortex, and the recorded signals were band-pass filtered in the alpha frequency band $(8-13 \mathrm{~Hz})$. They did not find any correlation between activity in the frontal cortex and curiosity. It was therefore of interest to extract information from multiple electrodes (here 61) and frequency bands (here ten).

The FBCSP+LDA algorithm, which won the fifth international BCI competition [26], works as follows: first, the training phase consists of optimally identifying and extracting both spatial and spectral features. For the spectral dimension, i.e., the frequency bands, EEG signals are filtered into ten $4 \mathrm{~Hz}-$ wide frequency bands (in $1-4 \mathrm{~Hz}, 4-8 \mathrm{~Hz}, \ldots, 36-40 \mathrm{~Hz}$ ) as in [26]. For each band, the band-pass filtered EEG trials are used. Then spatial filters are built for each band using the Common Spatial Pattern (CSP) algorithm [28], which optimizes the EEG signal-to-noise ratio: the variance of spatially filtered signals is maximized for one class and minimized for the other class. In our study, 4 CSP filters (2 pairs) have been optimized for each frequency band, resulting in 40 features (4 CSP filters $* 10$ frequency bands). From those 40 features, 4 were selected using the maximum Relevance Minimum Redundancy (mRMR) feature selection algorithm [29] to train an LDA classifier to discriminate curious from non-curious trials.

The second algorithm, FBTSC, [27] represents EEG signals as covariance matrices and manipulates them with Riemannian geometry [30]. Here, each trial is first band-pass filtered in the same ten $4 \mathrm{~Hz}$-wide frequency bands we used with the FBCSP $(1-4 \mathrm{~Hz}, 4-8 \mathrm{~Hz}, \ldots, 36-40 \mathrm{~Hz})$. To design the classifier, the average spatial covariance matrix for each class (curious and non-curious) is computed for each frequency band, and all covariance matrices are then projected in tangent space at the point defined as the matrices mean. We then used the softmax function-based probabilistic output of a Logistic Regression (LR) that has been trained in the tangent space, to determine probabilities of belonging to each class. Since we have two classes and a bank of ten frequency bands, 10 pairs of probabilities were computed. From these pairs of probabilities, the four most discriminant were selected using mRMR on the training set, and then multiplied together in order to obtain 2 final probabilities, i.e., one for the "curious" class and one for the "non-curious" class. The class assigned to a test EEG trial was decided according to the highest probability.

4) Evaluation: We assessed the performances of both algorithms using a within-participant study with five-fold stratified Cross-Validation. This means the data from each participant was divided into five parts: four parts were used for training the classifier and the fifth one for testing the resulting curiosity classifier for that participant. This process was repeated five times, with each part used exactly once as the testing set.

\section{RESULTS}

The F1-score, which is the weighted average of the precision and the recall, for each classifier and each time window length are reported in Figure 5. As a reference, the statistical chance levels using [31] was estimated at 51.59\% (100 trials per participants on average, 27 participants).

The boxplot of the performances obtained by each algorithm with the different time window lengths are reported in Figure 6 . We performed a 2-way ANOVA with repeated measures to evaluate the performance of the factor Time Window according to the factor Algorithm (FBCSP+LDA vs FBTSC). Note that we checked the data sphericity, as well as the normality, and used Greenhouse-Geisser (GG) correction in ANOVA if needed. The ANOVA revealed a main effect of Time 


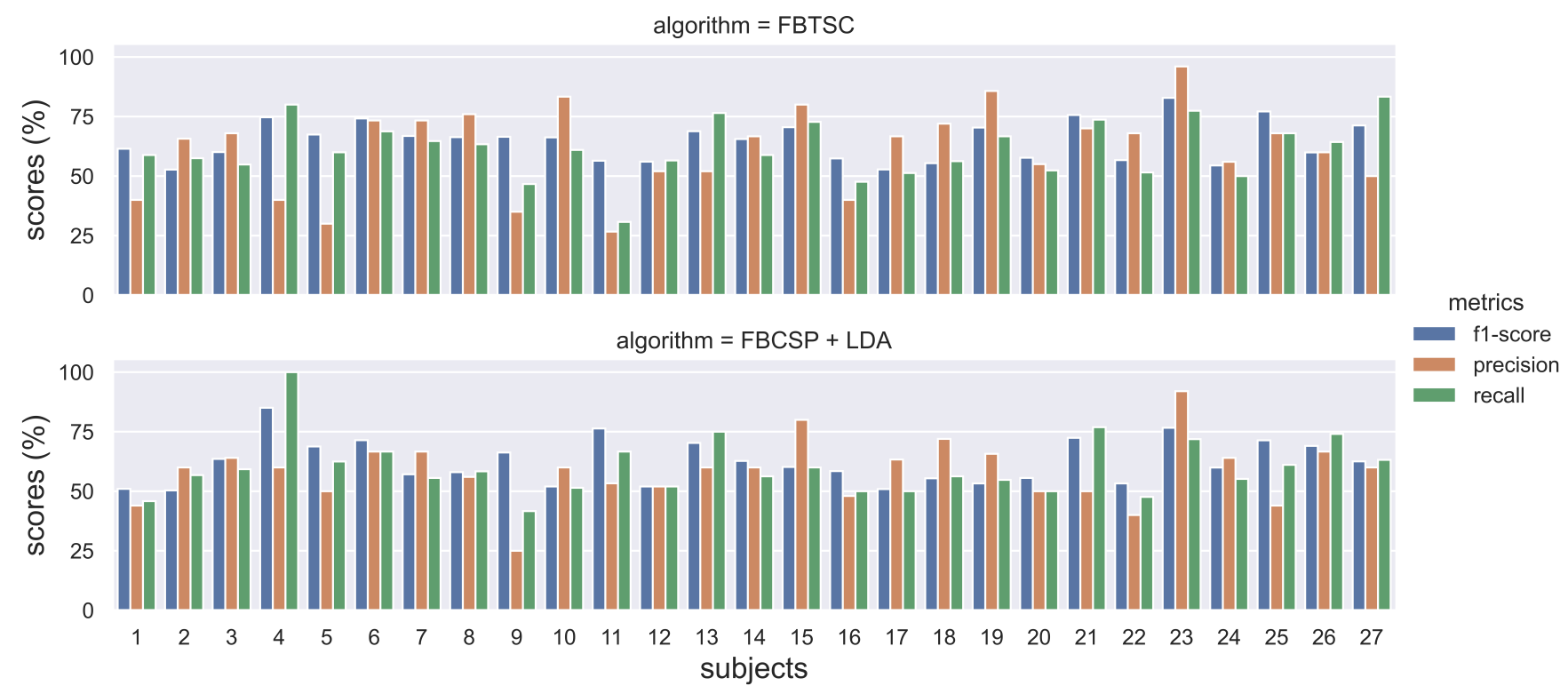

Fig. 4. F1 score, precision and recall for the 4-seconds time window length, for each subject and for each algorithm.

\begin{tabular}{|l|r|r|r|r|r|}
\hline & \multicolumn{1}{|c|}{$\mathbf{~ s e c}$} & $\mathbf{2 ~ s e c}$ & $\mathbf{3 ~ s e c}$ & $\mathbf{4 ~ s e c}$ & $\mathbf{5 ~ s e c}$ \\
\hline FBCSP+LDA & 53.40 & 57.34 & 59.87 & 60.93 & 59.82 \\
\hline FBTSC & 57.36 & 55.05 & 62.22 & 63.09 & 62.91 \\
\hline
\end{tabular}

Fig. 5. Average classification performances (F1-score) across participants obtained by each algorithm with the different time window lengths.

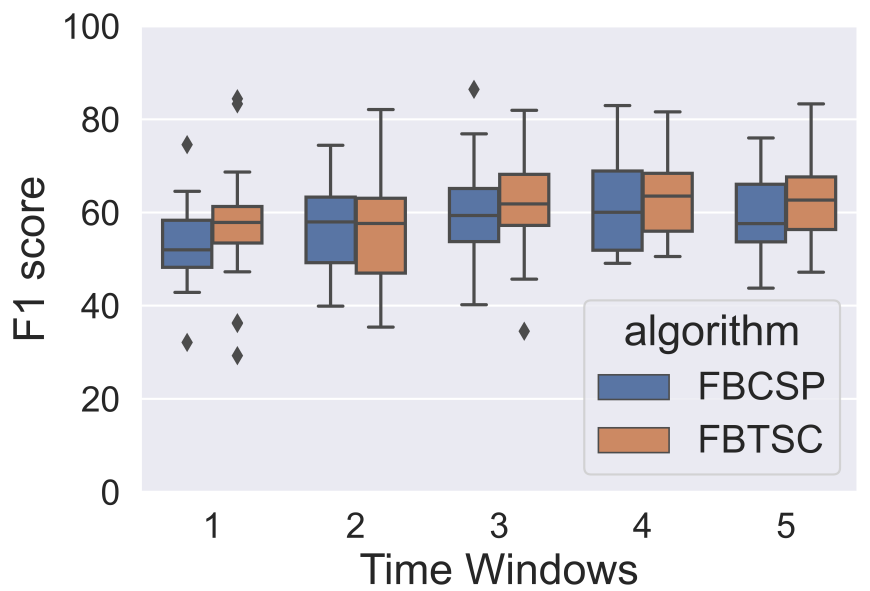

Fig. 6. F1-score for the different time window lengths, for each algorithm.

Window $[\mathrm{GG}(1,27)=0.825, \mathrm{p}=0.00004]$, but not for Algorithm $[\mathrm{F}(1,27)=1.588, \mathrm{p}=0.218859]$ nor for Time Window $*$ Algorithm [GG(1,27) $=0.643, \mathrm{p}=0.285987]$.

Post-hoc analyses results using Student t-test with False Discovery Rate (FDR) corrections showed significant differences between the 1-second time window and 3, 4, 5-seconds time windows when using the FBCSP+LDA $\left[\right.$ perf $_{1-s e c}=$ $53.4 \%$, perf $_{3-\text { secs }}=59.86 \% ; p \leq 0.05$, perf $_{4-\text { secs }}=60.93 \%$; $p \leq 0.05$, perf $\left._{5-\text { secs }}=59.81 \% ; p \leq 0.05\right]$. The same method showed significant differences between the 2-second time window and $3,4,5$-seconds time windows when using the FBTSC $\left[\right.$ perf $_{2-s e c}=55.05 \%$, perf ${ }_{3-\text { secs }}=59.86 \%$; $p \leq 0.05$, perf $_{4-\text { secs }}=63.08 \% ; p \leq 0.05$, perf $_{5-\text { secs }}=$ $62.91 \% ; p \leq 0.05]$. The maximum performances for both FBCSP+LDA $\left[\operatorname{perf}_{F B C S P+L D A}=60.93 \%\right.$, chance level $=$ $51.59 \% ; p \leq 0.05]$ and FBTSC $[$ perf $F B T S C=63.08 \%$, chance level $=51.59 \% ; p \leq 0.05]$ significantly outperformed the chance level for the 4-seconds time window.

Figure 4 shows the F1-score, precision and recall for each participant and algorithm, with the 4-seconds time window length, i.e., the time window with which both classification algorithms obtained the best performances. Still using the 4seconds time window length, we also studied the percentage of time that each frequency band was selected (by mRMR) by each algorithm, as reported in Figure 7. Those results show that the ML algorithms mainly used 4 frequency bands-i.e., delta $(1-4 \mathrm{~Hz})$, theta $(4-8 \mathrm{~Hz})$, alpha $(8-12 \mathrm{~Hz})$ and low beta $(12-$ $16 \mathrm{~Hz}$ ) - to classify states of epistemic curiosity.

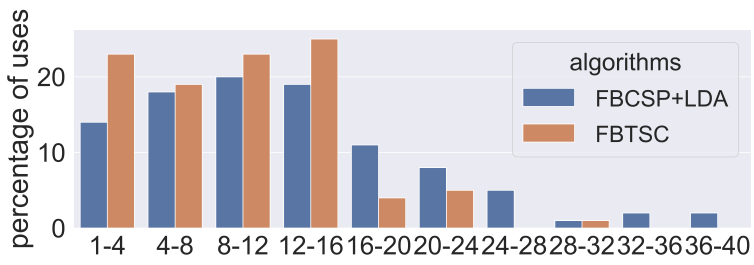

Fig. 7. Percentage of time that each frequency band was selected by each algorithm, with the 4-seconds time window length.

\section{Discussion, CONCLUSION AND FutURE WORK}

In this paper, we conducted an experiment aimed at collecting EEG signals during states of curiosity that were triggered using chains of trivia questions, and using ML to distinguish curious vs non-curious states. We used two ML 
algorithms, i.e., the FBCSP+LDA and FBTSC, to classify EEG signals at five different time-window lengths. The best results were obtained with the FBTSC, reaching about $62 \%$ of F1score for the 3, 4 and 5s time-windows (respectively 62.22, 63.09 and $62.90 \%$ ), significantly outperforming the chance level $(51.59 \%)$. Results in those 3 time-window lengths also significantly outperformed the results in the 2 s time window $(55.05 \%)$, but not the ones in the 1s time window $(57.36 \%)$. The FBCSP+LDA reached an F1-score of 59\% for the 3, 4 and 5s time windows, with respectively 59.90, 60.09 and $59.81 \%$, significantly outperforming both the chance level $(51.59 \%)$ and the results in the $1 \mathrm{~s}$ time window $(53.39 \%)$, but not the ones in the 2 s time window $(57.34 \%)$. Overall, results indicate that both algorithms obtained better performances in the 3-to5 s time windows, suggesting a minimum time of 3 seconds to go towards curiosity state estimation based on EEG signals. Moreover, ML algorithms mostly used a range of frequency bands from delta to low beta in order to classify states of epistemic curiosity, suggesting variations of EEG activity in the low frequencies during states of epistemic curiosity.

As future work, we will explore ways to measure curiosity states through EEG without participant-dependent calibration. While trivia questions were used as a trigger of curiosity, new tools (e.g., social robots [9], video games) or stimuli (e.g., videos of magic tricks) could be used in future experiments. We will also perform deeper neurophysiological analysis to identify the EEG sensors and sources mostly modulated by curiosity levels. For example, so far, results suggested that most of the information for discriminating curiosity levels are found in theta, alpha and low beta. These frequency bands are similar to the ones used to estimate levels of workload and levels of engagement [32]. This is interesting given that both states share some common characteristics with curiosity, such as implications in long-term memory improvements. Their similarities and differences would thus need to be further studied. Further analyses can also be done to compare our results against those obtained with fMRI [19], [21], in order to gain a better understanding of the neurological markers underlying curiosity states. Finally, analyses of physiological signals, e.g., breathing, electrodermal activity or heart rate, could be added to enhance curiosity decoding [33].

\section{ACKNOWLEDGMENT}

This work received support from the European ResearchCouncil (grant ERC-2016-STG-714567) and the BordeauxWaterloo Joint Grant 2019-2021.

\section{REFERENCES}

[1] P.-Y. Oudeyer, J. Gottlieb, and M. Lopes. Intrinsic motivation, curiosity, and learning: Theory and applications in educational technologies. Progress in Brain Research, 229:257-284, 2016.

[2] C. Kidd and B. Y. Hayden. The psychology and neuroscience of curiosity. Neuron, 88(3):449-460, 2015.

[3] J. Gottlieb and P.-Y. Oudeyer. Towards a neuroscience of active sampling and curiosity. Nature Reviews Neuroscience, 19(12):758-770, 2018.

[4] P.-Y. Oudeyer, F. Kaplan, and V. V. Hafner. Intrinsic motivation systems for autonomous mental development. IEEE Trans. Evol. Comp., 2007.

[5] G. Gordon, C. Breazeal, and S. Engel. Can children catch curiosity from a social robot? Proc. IEEE ICHRI, pages 91-98, 2015.
[6] R. Tieben, T. Bekker, and B. Schouten. Curiosity and interaction: making people curious through interactive systems. In Proc. BCS-HCI, 2011.

[7] E. Law, M. Yin, J. Goh, K. Chen, M. A Terry, and K. Z. Gajos. Curiosity killed the cat, but makes crowdwork better. CHI, 2016.

[8] S. Freeman, S. L. Eddy, M. McDonough, N. Okoroafor, H. Jordt, and M. P. Wenderoth. Active learning increases student performance in science, engineering, and mathematics. Academy of Sciences, 111, 2014.

[9] J. Ceha, N. Chhibber, J. Goh, C. McDonald, P.-Y. Oudeyer, D. Kulić, and E. Law. Expression of curiosity in social robots: Design, perception, and effects on behaviour. CHI, 2019.

[10] M. Clerc, L. Bougrain, and F. Lotte. Brain-Computer Interfaces 1: foundations and methods. ISTE-Wiley, 2016.

[11] T.O. Zander and C. Kothe. Towards passive brain-computer interfaces: applying brain-computer interface technology to human-machine systems in general. JNE, 2011.

[12] C. Jeunet, B. N'Kaoua, and F. Lotte. Towards a cognitive model of MI-BCI user training. In International Graz BCI Conference , 2017.

[13] M. J Gruber and A. Valji. 16 Curiosity and Learning. 2019.

[14] D. E. Berlyne. A theory of human curiosity. British Journal of Psychology, 1954.

[15] George Loewenstein. The psychology of curiosity: A review and reinterpretation. Psychological Bulletin, 1994.

[16] P. Mussel. Epistemic curiosity and related constructs: Lacking evidence of discriminant validity. Personality and Individual Differences, 2010.

[17] J. A Litman. Encyclopedia of the Sciences of Learning. Encyclopedia of the Sciences of Learning, (December), 2012.

[18] G. Brod and J. Breitwieser. Lighting the wick in the candle of learning: generating a prediction stimulates curiosity. Science of Learning, 2019

[19] M. Kang, M. Hsu, I. Krajbich, G. Loewenstein, S. M. McClure, J. Wang, and C. Camerer. The wick in the candle of learning: Epistemic curiosity activates reward circuitry and enhances memory. Psych. sci., 2009.

[20] R. A. Adcock, A. Thangavel, S. Whitfield-Gabrieli, B. Knutson, and John D.E. Gabrieli. Reward-Motivated Learning: Mesolimbic Activation Precedes Memory Formation. Neuron, 50(3):507-517, 2006.

[21] M. J. Gruber, B. D. Gelman, and C. Ranganath. States of Curiosity Modulate Hippocampus-Dependent Learning via the Dopaminergic Circuit. Neuron, 84(2):486-496, 2014.

[22] G. Lima. Curiosity, frontal EEG asymmetry, and learning. 41st Meeting of the CogSci Society, pages 2161-2165, 2019.

[23] Y. Renard, F. Lotte, G. Gibert, M. Congedo, and A. Lécuyer. OpenViBE: An open-source software platform to design, test, and use braincomputer interfaces in real and virtual environments. Presence, 2010.

[24] J. M. Hektner and M. Csikszentmihalyi. A Longitudinal Exploration of Flow and Intrinsic Motivation in Adolescents. American Educational Research Association, page 31, 1996.

[25] F. Lotte, L. Bougrain, A. Cichocki, M. Clerc, M. Congedo, A. Rakotomamonjy, and F. Yger. A Review of Classification Algorithms for EEG-based Brain-Computer Interfaces: A 10-year Update. JNE, 2018.

[26] K.K. Ang, Z.Y. Chin, C.C. Wang, C.T. Guan, and H.H. Zhang. Filter bank common spatial pattern algorithm on BCI competition IV datasets 2a and 2b. Frontiers in Neuroscience, 2012.

[27] A. Appriou, A. Cichocki, and F. Lotte. Modern machine learning algorithms to classify cognitive and affective states from electroencephalography signals. IEEE SMC Magazine, pages 1-8, 2020.

[28] B. Blankertz, R. Tomioka, S. Lemm, M. Kawanabe, and K.-R. Müller Optimizing spatial filters for robust EEG single-trial analysis. IEEE Sig Proc Magazine, 2008.

[29] H. Peng, F. Long, and C. Ding. Feature Selection Based On Mutual Information: Criteria of Max-Dependency,Max-Relevance, and MinRedundancy. IEEE Trans Pattern Anal Mach Intell, 27, 2005.

[30] F. Yger, M. Berar, and F. Lotte. Riemannian approaches in BrainComputer Interfaces: a review. IEEE TNSRE, 25(10), 2016.

[31] E. Combrisson and K. Jerbi. Exceeding chance level by chance: The caveat of theoretical chance levels in brain signal classification and statistical assessment of decoding accuracy. J Neur. Meth., 2015.

[32] F. Dehais, A. Lafont, R. Roy, and S. Fairclough. A Neuroergonomics Approach to Mental Workload, Engagement and Human Performance. Frontiers in Neuroscience, 2020.

[33] R. Knapp, J. Kim, and E. Andre. Physiological signals and their use in augmenting emotion recognition for human-machine interaction. Emotion-oriented systems, pages 133-159, 102011. 